\title{
Erneute Antiangiogenese wirksam
}

\author{
Auch wenn ein metastasiertes kolorektales Karzinom \\ (CRC) bereits antiangiogenetisch vorbehandelt ist, kann \\ eine erneute Antiangiogenese wirksam sein, wenn das \\ Therapieprinzip besonders effektiv ist.
}

Aflibercept ist ein gezielt entwickeltes Fusionsprotein, das nicht nur den vaskulären endothelialen Wachstumsfaktor VEGF-A abfängt, sondern auch VEGF-B und den Placenta growth Factor (PlGF), zwei weitere, die Tumorangiogenese stimulierende, Wachstumsfaktoren. Der Wirkstoff ermöglicht so eine umfassendere angiogene Blockade als dies bislang möglich war, erläuterte Ralf-Dieter Hofheinz, Mannheim. So bietet die Therapie mit Aflibercept Patienten mit bereits vorbehandeltem, metastasiertem kolorektalem Karzinom noch einmal die Chance auf einen signifikanten Überlebensvorteil. In einer Phase-III-Studie reduzierte die zusätzliche Behandlung mit Aflibercept zur Chemotherapie (FOLFIRI) das Sterberisiko der bereits vorbehandelten Patienten mit metastasiertem CRC im Vergleich zur alleinigen Chemotherapie um knapp 20\% (HR 0,817; $\mathrm{p}=0,0032$ ) [Tabernero J et al. Eur J Cancer. 2011;47(2):6LBA]. Der signifikante Überlebensvorteil zeigte sich auch bei den etwa $30 \%$ mit Bevacizumab vorbehandelten Patienten, betonte Hofheinz. Er hob zudem die für eine Zweitlinientherapie vergleichsweise hohe und gegenüber dem Kontrollarm signifikant erhöhte Ansprechrate von fast $20 \%$ (19,8 vs. $11,1 \%$; p $=0,0001$ ) hervor. Die mit dem Tumoransprechen einhergehende Reduktion des Tumorvolumens lindere auch die durch den Tumor ausgelösten Beschwerden. Gleichzeitig wird Aflibercept laut Hofheinz in der Regel gut vertragen.

Birgit-Kristin Pohlmann

Pressekonferenz „Expertise CRC" am 19. April 2012 in Königsstein; Veranstalter: Sanofi Aventis

\section{TKIs unterschiedlich effektiv}

\section{Der Tyrosinkinase-Inhibitor (TKI) Imatinib hat die Prognose von CML- Patienten dramatisch verbessert. Doch es geht noch spezifischer.}

Nach 3-Jahres-Daten der Phase-III-Studie ENESTnd induziert Nilotinib (Tasigna $\left.{ }^{\circledR}\right)$ eine doppelt so hohe Rate tiefer Remissionen $\mathrm{MR}_{4,5}$ wie Imatinib, berichtete Oliver Ottmann, Frankfurt/Main. In der dreiarmigen Studie erhielten 846 Patienten mit neu diagnostizierter CML in chronischer Phase randomisiert entweder Imatinib (400 mg/Tag) oder Nilotinib (300 mg 2x/ Tag oder $400 \mathrm{mg} 2 \mathrm{x} / \mathrm{Tag}$ ). Nach 3-jähriger Therapie waren die Raten einer guten molekularen Remission (MMR) in den Nilotinib-Armen signifikant höher als mit Imatinib (73\% mit $300 \mathrm{mg}$ vs. $70 \%$ mit $400 \mathrm{mg}$ vs. $53 \%$; p < 0,0001) [Clark F et al. EHA. 2012;Abstr. 0583]. Zudem erreichten 32 bzw. $28 \%$ der mit Nilotinib behandel- ten Patienten eine tiefe $\mathrm{MR}_{4,5}$-Remission, aber nur $15 \%$ der Patienten im Vergleichsarm ( $p<0,0001$ bzw. 0,003). Dabei kam es unter Nilotinib signifikant seltener zu einer akzelerierten Phase und Blastenkrise. Eine tiefe Remission zahlt sich aus: Patienten, deren BCR-ABL-Level nach drei Monaten $\leq 1 \%$ lag, hatten nach drei Jahren eine höhere Chance für das Erreichen einer MMR und $\mathrm{MR}_{4,5}$ sowie ein besseres Überleben [Hochhaus A et al. EHA. 2012; Abstr. 0584]. 43 mit Imatinib behandelte Patienten erreichten diesen prognostisch wichtigen Wert, in den Nilotinib-Armen 145.

Katharina Arnheim

Pressekonferenz "Meet the Expert: Mit Nilotinib neuen Standard in der CML-Therapie setzen" sowie Sitzung "First Line Trials in Chronic Myeloid Leukemia". 17th Congress of the European Hematology Association (EHA), 14.-17. Juni 2012 in Amsterdam; Veranstalter: Novartis Pharma

\section{Länger leben trotz Hautkrebs}

Der BRAF-Inhibitor Vemurafenib (Zelboraf ${ }^{\circledast}$ ) verbessert auch über eine längere Beobachtungszeit das Gesamtüberleben von Patienten mit einem nicht resezierbaren oder metastasierten Melanom und einer BRAF-V600-Mutation. Nach den auf dem diesjährigen ASCO-Kongress vorgestellten aktualisierten Ergebnissen der Phase-III-Studie BRIM-3 (BRAF-Inhibitor in Melanoma) lag das mediane Gesamtüberleben unter Vemurafenib mit 13,6 Monaten signifikant höher als unter Dacarbazin (9,7 Monate; HR 0,70; $\mathrm{p}=0,0008$ ) [Chapman $\mathrm{P}$ et al. J Clin Oncol. 2012;30(Suppl):Abstr 8502]. Das Sterberisiko war damit um $30 \%$ verringert. Nach zwölf Monaten lebten im Vemurafenib-Arm noch 56\% der Patienten gegenüber $44 \%$ im Chemotherapie-Arm. Zum Analysezeitpunkt lag das mediane progressionsfreie Überleben im Vemurafenib-Arm bei 6,9 Monaten auch signifikant über dem im Vergleichsarm (1,6 Monate; HR 0,38; p $<0,0001)$. Als unerwünschte Wirkungen traten neben Hautausschlägen und Juckreiz sowohl eine erhöhte Photosensitivität, Fatigue und Gelenkschmerzen als auch Hautläsionen wie Keratoakanthome oder Plattenepithelkarzinome auf. Derartige Hautläsionen sind durch regelmäßige Überwachung und Exzisionen bei Auftreten zu kontrollieren und stellten in der Studie keinen Grund für einen Therapieabbruch dar.

Ein neues Therapieprinzip könnte die Behandlungsmöglichkeiten beim fortgeschrittenen Basalzellkarzinom (BCC) erweitern. Vismodegib hemmt spezifisch den bei $90 \%$ der BCC aktivierten Hedgehog-Signalweg. Die Substanz ist bereits in den USA zugelassen. Grundlage dafür war die einarmige, multizentrische, offene Zulassungsstudie ERIVANCE BCC mit 71 Patienten mit lokal fortgeschrittenem BCC und 33 Patienten mit metastasiertem BCC. $42,9 \%$ der Patienten mit lokal fortgeschrittenem und 30,3\% der Patienten mit metastasiertem BCC sprachen auf die Therapie an [Dirix L et al. EMCC. 2011;Abstract 1BA]. Die Zulassung für die Europäische Union (EU) wurde im Dezember 2011 beantragt.

Nach Informationen von Roche 